\title{
Die Begegnung am Brunnen (Joh 4.4-42) im Licht der „Schrift“: Überlegungen zu den Samaritanern im Johannesevangelium*
}

\author{
HANS FÖRSTER \\ Institut für Neutestamentliche Wissenschaft, Evangelisch-Theologische Fakultät, \\ Universität Wien, Schenkenstr.8-10,A-1010Wien.Email: hans.foerster@univie.ac.at
}

\begin{abstract}
Within the narrative of John 4.4-42 (the Samaritan woman at the well) the fact is mentioned that 'Jacob's well' had been given to his son Joseph (John 4.5). By calling Jacob 'our father' (John 4.12), the woman claims for the Samaritans a direct lineage from Jacob via Joseph. The Samaritans are designated as 'children of Israel' and members of the tribes of Ephraim and Manasseh (i.e. the 'house of Joseph'). The genealogy is embedded in a motif occurring in Scripture (the encounter at the well). Thus, it is questionable whether the passage of the woman at the well can primarily be seen as 'mission among gentiles'. The narrative focus of the passage seems to point to a mission among the 'children of Israel'.
\end{abstract}

Keywords: Samaritans, the Gospel of John, Judaism, Hellenism

\section{Einleitung}

Das Johannesevangelium berichtet von einer Begegnung zwischen Jesus und einer namenlosen samaritanischen Frau ${ }^{1}$ am Jakobsbrunnen. Diese Begegnung, die als Anspielung auf ein Motiv der „Schrift“ verstanden werden kann, ${ }^{2}$ hat die exegetische Literatur stark befruchtet. Der Verfasser des Johannesevangeliums scheint bei seiner Konstruktion der Erzählung auf der

* Die Fragestellung entstand aus einem Forschungsprojekt (FWF-Projekt P24649 gefördert vom Österreichischen Wissenschaftsfonds/funded by the Austrian Science Fund; Frau Mag.a Ulrike Swoboda danke ich für Mitarbeit im Rahmen des Forschungsprojekts); eine intensive Auseinandersetzung mit der Thematik ermöglichte ein Forschungsaufenthalt in Berlin aufgrund eines Friedrich-Wilhelm-Bessel-Preises der Alexander von Humboldt-Stiftung.

1 Vgl. einleitend zu den Samaritanern L. D. Matassa, „Art. Samaritans. History. Samaritan Origins“, Encyclopaedia Judaica, Bd. XVII $\left(2007^{2}\right)$ 719-23.

2 R. Zimmermann, „From a Jewish Man to the Savior of the World: Narrative and Symbols Forming a Step by Step Christology in John 4,1-42", Studies in the Gospel of John and its Christology: Festschrift Gilbert van Belle (BEThL 265; Hg. J. Verheyden/G. van Oyen/M. Labahn/R. Bieringer; Leuven u.a.: Peeters, 2014) 99-118, hier 105. 
narrativen Ebene Aussagen über das Verhältnis von Juden und Samaritanern eingebunden zu haben, die vor dem Hintergrund der „Schrift” eine tiefere Bedeutung erhalten. ${ }^{3}$

\section{Traditionelle Deutungen der Perikope}

Von der Deutung der Samaritanerin (und damit eben gerade auch von der Beurteilung des Verhältnisses von Juden und Samaritanern) hängt ab, ob die vorliegende Stelle als speziell für die Samaritaner-Mission verfasst bzw. aus dieser entstanden oder als eine „Sendung Jesu in die Welt" ${ }^{\text {“4 }}$ gedeutet wird. Die Samaritanerin wird teilweise als gesellschaftliche „Außenseiterin“5 ${ }^{\text {“ }}$ konstruiert. Allerdings hat sie ein ausreichendes Ansehen, dass aufgrund ihres Zeugnisses Samaritaner zum Glauben kommen (Joh 4.39). Dies würde gegen die Zuschreibung einer „Außenseiter-Rolle“ sprechen. ${ }^{6}$ Und so kann dann diese Erzählung auch als „Ausdruck der hohen Wertschätzung [gewertet werden], die Frauen in den johanneischen Gemeinden offenkundig genossen: Die Samaritanerin ist die erste Missionarin im Buch (Maria Magdalena die letzte); überwältigt von Jesus, bringt sie viele Menschen ihrer Heimatstadt durch ihr Zeugnis (V. 39) zum Glauben an ihn. ${ }^{\text {"7 }}$

Als eine mögliche Interpretation der Erzählung und gleichzeitig als möglicher Sitz im Leben wird eine christliche Mission unter Samaritanern vorgeschlagen. ${ }^{8}$ Nach Jürgen Zangenberg handelt es sich bei der vorliegenden Passage um eine „ätiologische Legende zur Begründung der Existenz samarischer Christen und paränetischen Appell zur Legitimation ihrer Aufnahme in den joh Gemeindeverband“. ${ }^{9}$ Diese These ist nach Martina Böhm „in ihrem ersten Teil

3 Y. Amit, Hidden Polemics in Biblical Narrative (Translated from the Hebrew by Jonathan Chipman) (BIS 25; Leiden u.a.: Brill, 2000) 94: „The claim of the presence of concealed polemics may serve as an opening for an endless number of interpretations, which may at times lead one far astray from the structured meaning of the text: that is, from exegesis based upon the straightforward meaning of the text."

4 Vgl. C. Dietzfelbinger, Das Evangelium nach Johannes (ZBK 4/1 und 4/2; Zürich: Theol. Verl., $2004^{2}$ ) 95; so auch H. Boers, Neither on this Mountain nor in Jerusalem: A Study of John 4 (SBL. MS 35; Atlanta: Scholars, 1988) 199.

5 U. Schnelle, Das Evangelium nach Johannes (ThHK.NT 4; Leipzig: Evang. Verlagsanst., 2009 ${ }^{4}$ ) 96 (Vergleich von Joh. 3.1-12 und 4.1-42).

6 U. Wilckens, Das Evangelium nach Johannes (NTD 4; Göttingen: Vandenhoeck \& Ruprecht, $\left.2000^{2}\right) 80$.

7 M. Theobald, Das Evangelium nach Johannes: Kapitel 1-12 (RNT; Regensburg: Pustet, 2009) 342.

8 Wilckens, Johannes, 80; Dietzfelbinger, Johannes, 127; vgl. auch Schnelle, Johannes, 95.

9 J. Zangenberg, Frühes Christentum in Samarien: Topographische und traditionsgeschichtliche Studien zu den Samarientexten im Johannesevangelium (TANZ 27; Tübingen: Francke, 1998) 173. 
plausibel, in ihrem zweiten aber so wohl kaum belegbar“. ${ }^{10}$ Die Perikope von der Samaritanerin wird bei einer solchen Interpretation häufig im Zusammenhang mit Apg 1.8 und 8.4-8 gesehen. Allerdings weist z. B. Raymond Brown ausdrücklich darauf hin, dass in der Apostelgeschichte nicht erwähnt wird, dass Jesus bereits vor den Aktivitäten des Philippus Anhänger in Samarien hatte. ${ }^{11}$

Eine Deutung der vorliegenden Passage als „Sendung in die Welt“, ${ }^{12}$ d. h. eine Mission „außerhalb des Judentums" , ${ }^{13}$ wird häufig auch durch die synoptischen Inter- bzw. Prätexte ${ }^{14}$ bestärkt. Dietzfelbinger weist darauf hin, dass die „Synoptiker ... keinen missionarischen Erfolg Jesu bei den Samaritanern ...“ kennen und dass erst „nachösterlich“ mit Verweis auf die Apostelgeschichte von einer Mission in Samarien gesprochen werden könne. ${ }^{15}$ So findet sich in Mt 10.5b ein Verbot, „die Stadt der Samariter“ zu besuchen. Die „Zwölf“ werden in Mt 10.5-6 von Jesus nicht zu den „Völkern“ und nicht zu den Samaritanern, sondern vielmehr zu den „verlorenen Schafen aus dem Hause Israel“ gesandt. Nach Mt 10.6 scheinen die Samaritaner nicht zu den verlorenen Schafen des Hauses Israel zu gehören. ${ }^{16}$ Auch in Lk 9.52-3 ist eine eher feindliche Haltung zwischen Jesus und den Samaritanern zu erkennen, ${ }^{17}$ eine Deutung, die man ebenfalls durch Joh $4.9 \mathrm{c}$ begründet sieht, ${ }^{18}$ wo in einem redaktionellen Kommentar die große Distanz zwischen Samaritanern und Juden betont zu werden scheint. ${ }^{19}$ Da dieser Teil des Verses in Codex Sinaiticus (1. Hd.), in Codex Bezae Cantabrigiensis und in einer Reihe lateinischer Handschriften fehlt, wird dies auch als eine nachträgliche Glosse gedeutet. ${ }^{20}$

Auch tritt teilweise die Frau in den Vordergrund der Auslegungen. Sie kann zum Typus des „Menschen an sich“ werden, der auf die Botschaft Jesu reagiert. ${ }^{21}$ Ferner kann auch die persönliche Situation der Frau in ihren Beziehungen - so

10 M. Böhm, Samarien und die Samaritai bei Lukas: Eine Studie zum religionshistorischen und traditionsgeschichtlichen Hintergrund der lukanischen Samarientexte und zu deren topographischer Verhaftung (WUNT 2/111; Tübingen: Mohr Siebeck, 1999) 144.

11 R. E. Brown, The Gospel according to John (I-XII) (AncB 29; Garden City: Double Day, 1966) 175. vgl. auch J. Gnilka, Johannesevangelium (NEB; Würzburg: Echter, 1983) 36.

12 Dietzfelbinger, Johannes, 95.

13 M. Hengel, Die johanneische Frage: Ein Lösungsversuch mit einem Beitrag zur Apokalypse von Jörg Frey (WUNT 67; Tübingen: Mohr Siebeck, 1993) 300.

14 E. Haenchen, Das Johannesevangelium: Ein Kommentar (Tübingen: Mohr Siebeck, 1980) 248.

15 Dietzfelbinger, Johannes, 95. Siehe auch Brown, John (I-XII), 175.

16 So bereits J. Bowman, „Samaritan Studies“, BJRL 40 (1958) 298-327, hier 301 Anm. 1.

17 Brown, John (I-XII), 175.

18 S. Schulz, Das Evangelium nach Johannes (NTD 4; Göttingen: Vandenhoeck \& Ruprecht, $\left.1987^{16}\right) 73$.

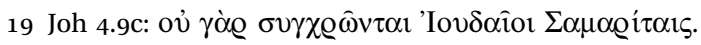

20 Siehe hierzu auch A. Link, „Was redest du mit ihr?“ Eine Studie zur Exegese-, Redaktions- und Theologiegeschichte von Joh 4,1-42 (BU 24; Regensburg: Pustet, 1992) 186-9.

21 R. V. Tasker, The Gospel according to St. John: An Introduction and Commentary (TNTC 4; London: Tyndale, 1972) 75. 
beispielsweise bei Johannes Calvin ${ }^{22}$ - in einer moralischen Weise gewertet werden. ${ }^{23}$ In diesem Kontext wird auch die Frage der Unwürdigkeit der Frau in Relation zu ihrer Zeugnisfähigkeit thematisiert. ${ }^{24}$ Vor dem Hintergrund dieser Deutungsmöglichkeiten ist nun $\mathrm{zu}$ fragen, ob möglicherweise andere Deutungen in dem Text angelegt sein könnten.

\section{Die Begegnung am Brunnen als Motiv der "Schrift“}

\subsection{Brunnen und keine Brautwahl}

Grundsätzlich sprachen Männer nicht mit unbekannten Frauen, sodass eine derartige Begegnung mit Aufnahme eines Dialogs als Motiv gesehen werden muss. ${ }^{25}$ Begegnungen an einem Brunnen können in der Schrift mit dem Motiv der Brautwahl bzw. Anbahnung einer Hochzeit verbunden sein. ${ }^{26}$ Dies gilt gerade für Texte, die dem Pentateuch zuzuordnen sind. ${ }^{27}$ Der Pentateuch wird von Juden und Samaritanern benutzt, sodass dieses Motiv sowohl für Samaritaner als auch für Juden eine Bedeutung hat. Deswegen wird in diesem Kontext auch von einer „,messianischen Brautwerbung' Jesu in Samarien“28 gesprochen.

Diese potentielle Brautwerbungsszene nimmt jedoch einen untypischen Verlauf. Eine Frau wird aufgefordert, ihren Mann zu rufen (Joh 4.16), und antwortet (formal korrekt), dass sie „keinen Mann habe“ (Joh 4.17a), bezeichnet sich also auf den ersten Blick als unverheiratet. Es scheint, dass mit Jesus und dieser Frau zwei unverheiratete (und damit heiratsfähige) Personen an diesem Brunnen

22 Vgl. M. Trebesius/H. C. Petersen, Johannes Calvins Auslegung des Johannes-Evangeliums (Johannes Calvins Auslegung der Heiligen Schrift Neue Reihe 14; Neukirchen-Vluyn: Neukirchener, 1964) 90: „Was war denn an dieser elenden Frau, daß der Sohn Gottes sie so unvermittelt aus einer Hure zu seiner Schülerin machte?"

23 Vgl. grundsätzlich L. Schottroff, „Die Samaritanerin am Brunnen“, Auf Israel hören: Sozialgeschichtliche Bibelauslegung (Hg. R. Jost/R. Kessler/C. M. Raisig; Luzern: Exodus, 1992) 115-32.

24 R. G. Maccini, „A Reassessment of the Woman at the Well in John 4 in Light of the Samaritan Context", JSNT 53 (1994) 35-46, hier 45-6.

25 Vgl. S. Schapdick, Auf dem Weg in den Konflikt: Exegetische Studien zum theologischen Profil der Erzählung vom Aufenthalt Jesu in Samarien (Joh 4,1-42) im Kontext des Johannesevangeliums (BBB 126; Berlin: Philo, 2000) 125.

26 Vgl. auch W. Rebell, Gemeinde als Gegenwelt: Zur soziologischen und didaktischen Funktion des Johannesevangeliums (BET 20; Frankfurt u.a.: Peter Lang, 1987) 189.

27 Vgl. J. D. Purvis, „The Fourth Gospel and the Samaritans“, NT 17 (1975) 161-98, hier 194.

28 Vgl. hierzu auch J. Frey, „,Gute' Samaritaner? Das neutestamentliche Bild der Samaritaner zwischen Juden, Christen und Paganen“, Die Samaritaner und die Bibel: Historische und literarische Wechselwirkungen zwischen biblischen und samaritanischen Traditionen (SJ 70/StSam 7; Hg. J. Frey/U. Schattner-Rieser/K. Schmid; Berlin: De Gruyter, 2012) 203-33, hier 227. 
zusammengekommen sind. Dieser Eindruck wird durch die Antwort Jesu zerstört (Joh 4.18): Fünf Männer habe sie gehabt und der, den sie jetzt habe, sei nicht ihr Mann. Deswegen habe sie wahr gesprochen.

In der Auslegung wird häufig die eheliche Situation der Frau moralisch wertend $^{29}$ thematisiert. Die fünf Männer der Samaritanerin wurden aber auch allegorisch als die fünf Sinne des Menschen oder die fünf ${ }^{30}$ Götzen der Samaritaner ${ }^{31}$ und der „Buhle Jahwe“ gedeutet. ${ }^{32}$ Vom Leser wird bei dieser Deutung des Textes einerseits eine vergleichsweise genaue Kenntnis von 2 Kön 17.24-41 (dort vor allem 30-1) ${ }^{33}$ und andererseits eine Unkenntnis des samaritanischen Pentateuch erwartet, der die Grundlage des „israelitischen“34 Monotheismus der Samaritaner mit ihrem Zentralheiligtum auf dem Garizim darstellt. ${ }^{35}$ Dies erweist die Deutung auf die „Götzen“ als problematisch und eröffnet die Frage nach dem Ort der Begegnung.

\subsection{Sychar beim Brunnen}

Der Brunnen hat bereits bei seiner ersten Erwähnung eine zentrale Stellung. Er scheint wichtiger zu sein als der $\mathrm{Ort}^{36}{ }^{36}$ in dessen Nähe er liegt,

29 Vgl. zur moralischen Wertung auch Rebell, Gemeinde als Gegenwelt, 190.

30 Frey weist darauf hin, dass in 2 Kön 17.30-1 sieben Götter genannt werden; bei Flavius Josephus, Ant. 9,288 entsprechen dann den fünf paganen Stämmen fünf Götter; dort findet sich auch die Bezeichnung Kutim für Samaritaner; Jos. Ant. 9,288: oi $\delta \dot{\varepsilon} \mu \varepsilon \tau 0 \varkappa \varkappa 1 \sigma \theta \varepsilon ́ v \tau \varepsilon \zeta$

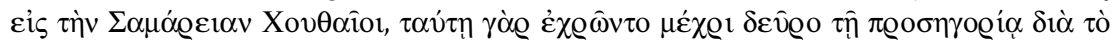

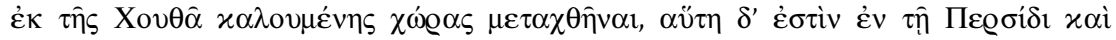

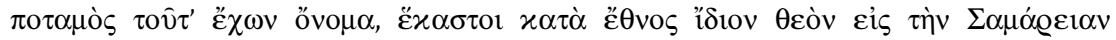

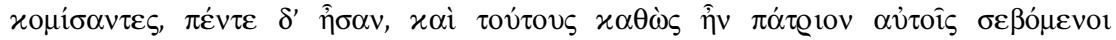

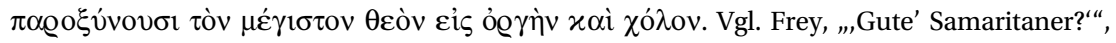
226-7; siehe auch R. Pummer, The Samaritans in Flavius Josephus (TSAJ 129, Tübingen: Mohr Siebeck, 2009) 281.

31 J. N. Day, The Woman at the Well: Interpretation of John 4:1-42 in Retrospect and Prospect (BIS 61; Leiden u.a.: Brill, 2002) 40.

32 Allerdings ist fraglich, ob dies tatsächlich „die“ jüdische Auffassung bezüglich der Samaritaner in der Antike darstellt, vgl. Haenchen, Johannesevangelium, 242: „Andere Ausleger zogen ebenfalls gegen den Sinn der Szene - eine allegorische Deutung vor: die Frau sei das samaritanische Volk, ihre fünf Männer jene fünf Götzen, welche die Samaritaner nach 2Kön 17,24ff. verehrten, und der Buhle sei Jahwe."

33 Vgl. z. B. S. Schorch, Die Vokale des Gesetzes: Die samaritanische Lesetradition als Textzeugin der Tora, Bd. I: Das Buch Genesis (BZAW 339; Berlin/New York: De Gruyter, 2004) 14.

34 Die Samaritaner bezeichnen sich selbst ja als „Israeliten“.

35 M. Kartveit, 'The Second Temple and the Temple of the Samaritans', Die Samaritaner und die Bibel, 67-80, hier 80.

36 H.-M. Schenke, „Jakobsbrunnen - Josephsgrab - Sychar“, ZDPV 84 (1968) 159-84, dort 167: „Und ganz merkwürdig ist nun, daß in V. 5 die Lage der Ortschaft Sychar nach dem

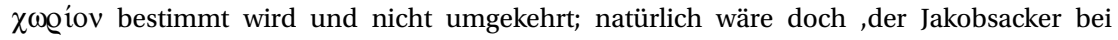
Sychar', aber es steht da ,Sychar beim Jakobsacker.' Zur Bedeutung vgl. Böhm, Samarien, 91: „Zureichende Aussagen über Größe und Bedeutung Sychars in hellenistisch-römischer 
befindet sich doch nach dem Wortlaut der Stelle der Ort „Sychar in der Nähe des Feldes, das Jakob seinem Sohn Josef gab. Und dort war der Brunnen“ (vgl. Joh 4.56a). Die relativ unbekannte Stadt Sychar begegnet auch in einigen anderen Quellen. ${ }^{37}$ Christliche Autoren der Antike scheinen für den Ort auf Joh 4.5 Bezug zu nehmen, ohne genaue Ortskenntnis zu besitzen. Eine Lokalisierung bei dem heutigen arabischen Dorf 'Askar scheint möglich, ${ }^{38}$ jedoch alles andere als sicher. ${ }^{39}$ In der syrischen Überlieferung wird der Name der Stadt zur bekannten Stadt Sichem korrigiert. ${ }^{40}$ Es ist also die Frage, worin die Bedeutung des "geschichtsträchtigen Ortes" genau besteht. ${ }^{41}$ Der Ort wird auch als „Nachfolgesiedlung des durch Johannes Hyrkan zerstörten Sichem“ gesehen. ${ }^{42}$ Auffällig ist, dass der „Jakobsbrunnen“ nur in christlichen Quellen begegnet. ${ }^{43}$

Die eher unbekannte Stadt Sychar betont also den Brunnen. Und dieser ist mit einer Genealogie verbunden: Das Feld wurde von Jakob seinem Sohn Josef gegeben. Und der Brunnen ist „die Quelle des Jakob“. Gleich an drei Stellen in der vorliegenden Erzählung von der Begegnung am Brunnen - aber dafür nur dort - begegnet der Erzvater Jakob im Johannesevangelium. ${ }^{44}$ Ferner handelt es sich um die einzige

Zeit zu treffen, ist aufgrund der spärlichen Informationslage kaum möglich.“ Dagegen jedoch Hengel, Die johanneische Frage, 279-80: ,Johannes ... weiß, daß das Dorf Sychar als damaliger Hauptort Samariens beim Jakobsbrunnen $(4,5)$ gegenüber dem heiligen Berg Garizim lag ...“

37 Siehe auch M. Hengel, „Der Historiker Lukas und die Geographie Palästinas in der Apostelgeschichte“, Martin Hengel: Studien zum Urchristentum. Kleine Schriften 6 (WUNT 234;

Hg. C. J. Thornton; Tübingen: Mohr Siebeck, 2008) 140-90, hier 185 (= ZDPV 99 (1983) 147-83). 38 Schenke, „Jakobsbrunnen - Josephsgrab - Sychar“, 181-4.

39 U. C. von Wahlde, „Archaeology and John's Gospel“, Jesus and Archaeology (Hg. J. H. Charlesworth; Grand Rapids: Eerdmans, 2006) 523-86, hier 558.

40 So auch Hieronymus, Quaest. in Gen 48,22 (Antin, Hieronymi Opera, Bd. I, 1, CChr.SL 72, 52,69) und Hieronymus, Ep. 108.13 (Hg. J. Labourt, Jerôme Lettres, Bd. v, 173,30-1): transivit Sichem, non ut plerique errantes legunt Sichar, quae nunc Neapolis appellatur. Für Hieronymus handelt es sich bei der Lesart Sychar für Sichem um einen Fehler im Johannesevangelium. Nach E. Noort, „Josua 24,28-31, Richter 2,6-9 und das Josuagrab: Gedanken zu einem Straßenschild“, Biblische Welten: FS für Martin Metzger zu seinem 65. Geburtstag (OBO 123; Hg. W. Zwickel; Freiburg/Göttingen: Universitätsverlag/Vandenhoeck \& Ruprecht, 1993) 109-30, handelt es sich bei der Angabe in Ep. 108 um einen Fehler des Hieronymus (vgl. ebd. 125).

41 K. Wengst, Das Johannesevangelium. 1. Teilband: Kapitel 1-10 (ThK.NT; Stuttgart: Kohlhammer; 2004²) 164-5.

42 Frey, „,Gute' Samaritaner?“, 221.

43 Siehe hierzu M. Hengel, „Das Johannesevangelium als Quelle für die Geschichte des antiken Judentums“, Jüdische Geschichte in hellenistisch-römischer Zeit: Wege der Forschung. Vom alten zum neuen Schürer (Schriften des Historischen Kollegs. Kolloquien 44; Hg. A. Oppenheimer; München: Oldenbourg, 1999) 41-73, hier 47 Anm. 26; vgl. auch B. Olsson, Structure and Meaning in the Fourth Gospel: A Text-Linguistic Analysis of John 2:1-11 and 4:1-42 (CB.NT 6; Lund: CWK Gleerup, 1974) 162-73.

44 Joh 4.5, 6, 12. 
Erwähnung des Stammvaters Josef im gesamten Johannesevangelium. ${ }^{45}$ Mit der wiederholten Erwähnung Jakobs wird der Blick geöffnet auf die Bedeutung des Erzvaters innerhalb der vorliegenden Erzählung. Dies ist im folgenden Abschnitt zu untersuchen.

\subsection{Jakob, Josef und die Samaritaner}

Am Beginn der Erzählung wird durch die Erwähnung des Umstandes, dass Jakob seinem Sohn Josef dieses Land gegeben hat, der Ort, an dem Jesus und die Frau einander begegnen, als zum Hause Josef ${ }^{46}$ gehörig identifiziert. ${ }^{47}$ Das Gebiet wird durch die Erwähnung des Stammvaters Josef für die Stämme Ephraim und Manasse reklamiert. ${ }^{48}$ Historisch war das Gebiet Samarien weitgehend identisch mit dem Stammesgebiet von Ephraim und Manasse. ${ }^{49}$ Nicht nur für Juden, sondern auch für Samaritaner gilt offensichtlich nach dieser Einleitung, dass sie vom Stammvater Jakob abstammen. Damit sind sie den zwölf Stämmen Israels zugeordnet und Teil des erwählten Volkes. ${ }^{50}$ In epigraphischen Zeugnissen bezeichnen sich Samaritaner als Israeliten. ${ }^{51}$

45 Als weiterer Stammvater wird Abraham (Joh 8.30-45) thematisiert. Dort finden sich in neun Versen insgesamt elf Belege des Namens Abraham (Joh 8.33, 37, 39, 40, 52, 53, 56, 57, 58).

46 Und es scheint, dass die Samaritaner selbst bei ihrer Zuordnung auf das „Haus Josef“ und nicht auf einen der beiden damit verbundenen Stämme verwiesen haben; vgl. H. G. Kippenberg, Garizim und Synagoge: Traditionsgeschichtliche Untersuchungen zur samaritanischen Religion der aramäischen Periode (RVV 30; Berlin/New York: De Gruyter, 1971) 255.

47 Siehe auch Kippenberg, Garizim und Synagoge, 255: „Josephs Grab, das von früh an bei Sichem lokalisiert ist, wird von den Samar. hoch verehrt. Zugleich gilt Joseph als der Stamm, zu dem sich die Samar. rechnen."

48 Nach Gen 48.5-7 beansprucht Jakob die beiden Söhne namens Manasse und Ephraim, die Josef mit seiner Frau Asenat, der Tochter Potiferas hatte (Gen 41.45), als seine eigenen Söhne. Diese werden die Stammväter zweier Stämme Israels. Es handelt sich bei dieser narrativen Beschreibung der Samaritaner als Nachfahren der beiden Söhne Josefs um die auch andernorts für das erste nachchristliche Jahrhundert bezeugte Selbstdefinition der

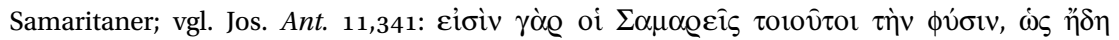

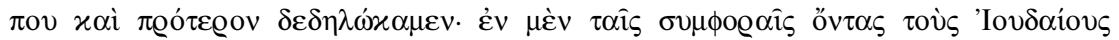

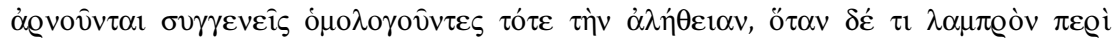

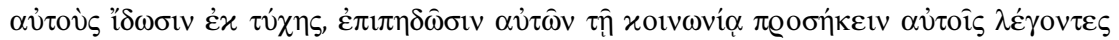

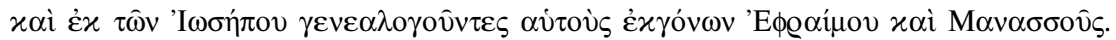
So auch z. B. Origenes, Comm. in Joh. 13,26,159.

49 Vgl. hierzu auch H. Donner, Geschichte des Volkes Israel und seiner Nachbarn in Grundzügen. Teil 1: Von den Anfängen bis zur Staatsreligion (ATD.E 4/1; Göttingen: Vandenhoeck \& Ruprecht, 2000 ${ }^{3}$ ) 158-60.

50 Vgl. Olsson, Structure and Meaning, 140-1: „Thus, according to the text, the Samaritans are the descendants of Jacob, descendants of Joseph etc. " Er zieht jedoch andere Konsequenzen (ebd. 278): „The confrontation underlying these texts would, therefore, not only include that between Judaism and Christianity, and that between Samaritanism and Christianity, but also reflect different ideas within the Church herself." Siehe ferner Schapdick, Auf dem Weg in den Konflikt, 130.

51 Das ist übrigens auch das Selbstverständnis der Samaritaner, wie es in den Inschriften auf Delos zum Ausdruck kommt; vgl. P. Bruneau, „Les Israélites de Délos et la juiverie 
Gegen diese Deutung könnte man die Frage der Frau anführen: „Wie, du bittest mich um etwas zu trinken, der du ein Jude bist und ich eine samaritanische Frau? ${ }^{\text {“52 }}$ Der redaktionelle Kommentar in $4.9 \mathrm{c}$ scheint eine strikte Trennung zwischen Juden und Samaritanern zu bestätigen. ${ }^{53}$ Der genaue Sinn des redaktio-

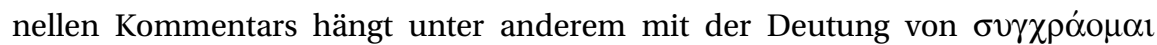
zusammen. Man könnte als Übersetzung - bei einer eher engen Deutung des Wortes - auch vorschlagen: „Denn die Juden gebrauchen nicht gemeinsam mit den Samaritanern. "54 "Nun fehlt freilich in Joh 4,9c ein direktes Objekt zum

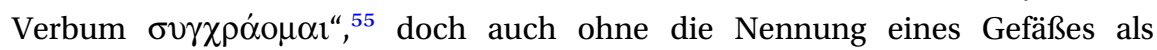
Objekt scheint das Wort nicht zwingend mit „Umgang pflegen“ zu übersetzen, da die Grundbedeutung "gemeinsam gebrauchen“ ist. ${ }^{56}$

An der vorliegenden Stelle wird bei dieser Deutung die Gefahr der rituellen Verunreinigung durch gemeinsamen Gebrauch von Gefäßen ${ }^{57}$ oder den Umgang mit Personen, die sich nicht an die strengen jüdischen Reinheitsgebote halten, vorausgesetzt; ${ }^{58}$ eine derartige Gefahr wird auch an einer anderen Stelle des Johannesevangeliums thematisiert. ${ }^{59}$ Bei diesem

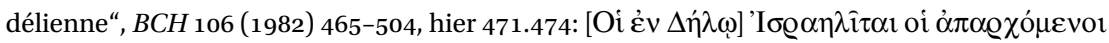

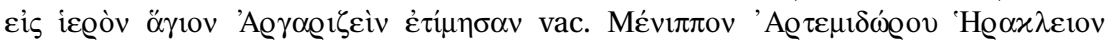

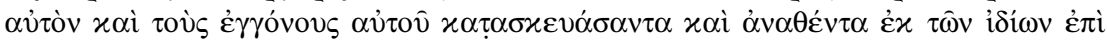

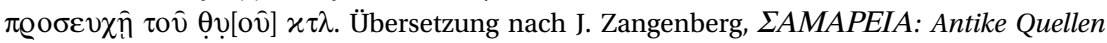
zur Geschichte und Kultur der Samaritaner in deutscher Übersetzung (Tübingen/Basel: Francke, 1994) 325: „[Die] Israeliten [auf Delos], die Opfer darbringen zum heiligen, geweihten Argarizim, ehrten [vac.] Menippos, Sohn des Artemidoros, aus Heraklion, ihn und seine Verwandten, der hergerichtet hat und geweiht hat aus eigenen Mitteln zur Anbetung Gottes [...].“ Vgl. auch Matassa, „Art. Samaritans“, 722: „The term Argarizein is the Greek rendering of the Hebrew Har Gerizim, that is, Mt. Gerizim ..." Siehe ferner M. Kartveit, The Origins of the Samaritans (VT.S 128; Leiden/Boston: Brill, 2009) 256. Sowie A. D. Crown, „Redating the Schism between the Judaeans and the Samaritans“, JBQ 82 (1991) 17-50, hier 19.

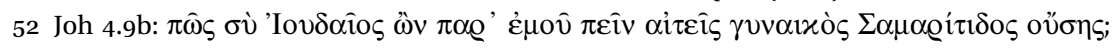

53 Vgl. Frey, „,Gute‘ Samaritaner?“, 224: „Durch die verwunderte Äußerung der Frau ... werden nicht nur Gender-Aspekte berührt, sondern auch ... die religiös-ethnische Distanz zwischen Juden und Samaritanern."

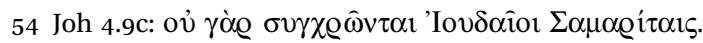

55 Frey, „,Gute' Samaritaner?", 225.

56 Diese Deutung des Begriffs $\sigma v \gamma \chi \varrho \alpha ́ o \mu \alpha 1$ wird auch durch die griechischen Papyrusurkunden bestätigt; vgl. F. G. Preisigke, Wörterbuch der griechischen Papyrusurkunden mit Einschluß der griechischen Inschriften, Aufschriften usw. aus Ägypten, Bd. II (Berlin: Selbstverlag, 1927) 501.

57 Vgl. auch S. Miller, „The Woman at the Well: John's Portrayal of the Samaritan Mission“, John, Jesus, and History, Bd. II: Aspects of Historicity in the Fourth Gospel (Hg. P. N. Anderson/F. Just/ T. Thatcher; Atlanta: SBL, 2009) 73-81, hier 75: „This translation is supported by the woman's comments that Jesus has no bucket, and he must rely on her water jar in order to drink some water (4:11)."

58 Zur grundsätzlichen Frage der Übertragung von Unreinheit, C. Hayes, „Art: Purity and Impurity, Ritual“, Encyclopaedia Judaica, Bd. xvi $\left(2007^{2}\right)$ 746-56. 
Verständnis von Joh 4.9c könnte man hinter dieser Frage der Frau (Joh 4.9b) eine Anspielung auf die zur Zeit Jesu existierende Auffassung finden, dass samaritanische Frauen als unrein galten ${ }^{60}$ und deswegen keine gemeinsame Nutzung von (potentiell unreinen) Gegenständen durch Juden und Samaritaner stattfand. ${ }^{61}$ Die fehlende Tischgemeinschaft führt selbstverständlich auch zu einer soziologischen Trennung von Juden und Samaritanern, ${ }^{62}$ die durchaus auch von jüdischen Rabbinern bewusst vorangetrieben wurde. ${ }^{63}$

Dass der Kommentar in Joh 4.9c in seiner traditionellen Deutung die Narrative der vorliegenden Passage durchbricht, wird durch den weiteren Verlauf des Gesprächs deutlich: In Vers 12 bemerkt die Frau: „Bist du denn mehr als unser

59 Vgl. Joh 18.28; J. J. Gibson, Peter between Jerusalem and Antioch: Peter, James and the Gentiles (WUNT 2/345; Tübingen: Mohr Siebeck, 2013) 114-15: „While there was no actual Jewish halakhah which declared the Gentiles to be impure they 'were treated as impure'. Thus do Jesus' accusers refuse to enter the Praetorium in Jerusalem, the residence of the Roman governor, ,so that they would not be defiled, but might eat the Passover' (John 18:28). "Siehe auch Frey, „,Gute' Samaritaner?“, 225-6, sowie P. Tomson, „Jewish Purity Laws as Viewed by the Church Fathers and by the Early Followers of Jesus“, Purity and Holiness: The Heritage of Leviticus (Jewish and Christian Perspectives Series 2; Hg. M. J. H. M Poorthuis/J. Schwartz; Leiden u.a.: Brill, 2000) 73-91, hier 83-4: „The other halakhah concerns the impurity of non-Jewish homes ... within the Land of Israel, which would prevent Jews from observing purity regulations from entering such homes."

60 Vgl. für eine Kritik dieser Ansicht Y. Magen, The Samaritans and the Good Samaritan (JSP 7; Jerusalem: Israel Antiquities Authority, 2008) 74: „One of the most difficult statements by the rabbis concerns the menstrual impurity of Samaritan women; it is especially puzzling in light of the laws of niddah."

61 D. Daube, „Jesus and the Samaritan Woman: the Meaning of $\sigma v \gamma \chi \varrho \alpha ́ o \mu \alpha \imath$ “, JBL 69 (1950) 137-47, hier 138. Die Auffassung, dass samaritanische Frauen grundsätzlich unrein seien, wurde zwischen 65 und $66 \mathrm{n}$. Chr. schriftlich fixiert; siehe $b$. Šabb. 16b (dort wird in der Erläuterung zur Stelle ausdrücklich die „heidnische Abstammung“ der Kutim betont, während gleichzeitig konzediert wird, dass diese in der Geschichte sowohl als Juden als auch als Nicht-Juden angesehen wurden); siehe ferner $b$. Šabb. 17a; vgl. $m$. Nid. 4,1-2; vgl. C. K. Barrett, The Gospel according to St. John: An Introduction with Commentary and Notes on the Greek Text (London: SPCK, $1978^{2}$ ) 232. Siehe auch Anm. 17. Vgl. hierzu Maccini, „Reassessment“, 39. Vgl. ferner T. Okure, The Johannine Approach to Mission (WUNT 2/31; Tübingen: Mohr, 1988) 95 Anm. 36, und zu m. Nid. 4,1 J. Hauptmann, Rereading the Mishnah (TSAJ 109; Tübingen: Mohr Siebeck, 2005) 122 Anm. 43. Zur Übertragung der Unreinheit vgl. ferner R. Biale, Women and Jewish Law: The Essential Texts, their History, and their Relevance for Today (New York: Schocken, 1984 = 1995) 151.

62 Gibson, Peter between Jerusalem and Antioch, 116: „There is also a tendency within Jewish writings to link Gentiles with idolatry, and that avoidance of the latter required avoidance of the former ... This connection between food and idolatry can also be seen in the second century вс Letter to Aristeas (128-143), which claims that the dietary laws were given by God specifically to keep the Jews separate from the other nations."

63 Magen, Samaritans, 75: „The rabbis were worried by the points of contact with the Samaritans, such as agricultural produce, tithes, and especially marriage. At all these junctures, the rabbis ruled against them, and thus erected a clear barrier to contact between Jews and Samaritans." 
Vater Jakob, der uns diesen Brunnen gegeben hat? Und er hat daraus getrunken und seine Kinder und sein Vieh. ${ }^{\text {"64 }}$ Die Samaritanerin bezeichnet mit dem Personalpronomen der ersten Person Plural im Genitiv - und damit ersetzt das Personalpronomen ein Possessivpronomen - ihr Verhältnis zu dem Stammvater Jakob. Es gibt grundsätzlich zwei Möglichkeiten, diese Zuordnung zu verstehen. Die naheliegende Möglichkeit ist, dass es sich um einen inklusiven Gebrauch des possessiv verwendeten Personalpronomens handelt: In diesem Fall würde die Frau zwischen ihrer eigenen Existenz als Samaritanerin und Jesu jüdischer Identität eine Brücke durch den gemeinsamen Vater Jakob schlagen. Er ist der Stammvater aller Israeliten und sowohl Juden als auch Samaritaner - d. h. Mitglieder der Stämme Ephraim und Manasse ${ }^{65}$ aus dem Hause Josef gehören zu einem Volk.

Eine andere, nicht völlig auszuschließende Deutungsmöglichkeit wäre ein exklusiver Gebrauch. ${ }^{66}$ In diesem Fall würde die Frau Jakob als Stammvater für sich reklamieren und dadurch in eben der Art, wie ein Teil der jüdischen Überlieferung den Samaritanern ihre Zugehörigkeit zum Volk Israel, den Juden ihre Zugehörigkeit zum Volk Israel absprechen und für sich allein die wahre Überlieferung reklamieren. Gegen diese Deutung lässt sich das Ende der wörtlichen Rede der Frau in Joh 4.12 anführen: Jakob hat zusammen mit seinen Kindern aus diesem Brunnen getrunken. Damit nimmt die Samaritanerin in ihren wörtlichen Reden einerseits auf die Aufteilung der Stammesgebiete durch Jakob Bezug: Das Land, auf dem dieser Brunnen liegt, wurde den Samaritanern gegeben. Andererseits betont sie, dass zwischen den Nachkommen Jakobs Gemeinschaft bestand: Jakob, seine Söhne und sein Vieh haben aus diesem Brunnen getrunken.

Grundsätzlich lassen sich diese zwei möglichen Deutungen des possessiv gebrauchten Personalpronomens mit der ambivalenten Situation zwischen Juden, Samaritanern und dem Selbstverständnis der Samaritaner in Verbindung bringen: Der Name „Samaritaner“ wurde aus dem zweiten Buch der Könige abgeleitet, wo er als Beispiel für eine Gruppe geführt wird, die Gott fürchtete, allerdings zusätzlich ihre Götzen anbetete. ${ }^{67}$ Nach dieser Darstellung handelt es sich um

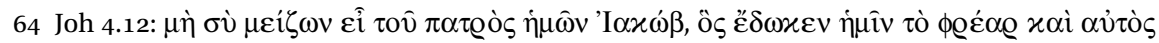

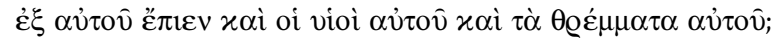

65 So ähnlich bereits E. D. Freed, „Samaritan Influence in the Gospel of John“, CBQ 30 (1968) 580-7, hier 581: „The Samaritans claim to be descendants from Ephraim and Manasseh. Cf., for example, Jn 4,12 ..."

66 Diese beiden Deutungen des Genitivs werden intratextuell durch Joh 4.20 bestätigt, da in diesem Text eindeutig ein exklusiver Gebrauch des possessiv verwendeten Personalpronomens zum

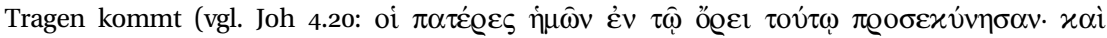

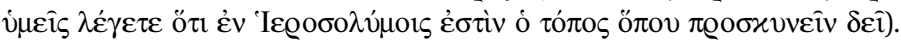

67 Siehe hierzu auch Schorch, Vokale des Gesetzes, 14. 
eine ethnisch dem Judentum zuzurechnende Gruppe, die jedoch den jüdischen Monotheismus um synkretistische Tendenzen erweitert hat. ${ }^{68}$

Möglicherweise sogar als direkte Reaktion auf diese Herleitung der Entstehung der Samaritaner in einer für die Samaritaner nicht kanonischen Schrift des Judentums wurde der Name der Glaubensgemeinschaft von den Samaritanern selbst nicht von dem von ihnen besiedelten Land, sondern vom hebräischen Wort שמר abgeleitet: Die Bedeutung des Namens kann bei dieser Etymologie mit „die Hüter des göttlichen Gesetzes“ angegeben werden. ${ }^{69}$ Ein relativ später Beleg für diese etymologische Deutung des Namens findet sich bei Epiphanius von Salamis. ${ }^{70}$ Der samaritanische Pentateuch hält selbstverständlich am jüdischen Monotheismus fest, sodass der in 2 Kön $17 \mathrm{zu}$ findende Vorwurf des Synkretismus diese Gemeinschaft nicht korrekt zu charakterisieren scheint. Gerade weil der samaritanische Pentateuch die normative Schrift der Gemeinschaft ist, ${ }^{71}$ stehen die unterstellten synkretistischen Tendenzen nicht in Einklang mit der theologischen Ausrichtung der Samaritaner. ${ }^{72}$

Die Betonung einer direkten Abstammung der Samaritaner vom Stammvater Jakob stellte eine Provokation dar. ${ }^{73}$ Trotzdem bezeichnet die unbekannte Samaritanerin am Brunnen sich und alle Samaritaner unwidersprochen als „Kinder Jakobs“. Gerade weil Jesus an anderer Stelle den Juden die Abrahamskindschaft abspricht, ${ }^{74}$ muss betont werden, dass die Berufung auf den gemeinsamen Vorfahren und Vater Jakob unwidersprochen bleibt. ${ }^{75}$ Nach Urban von Wahlde handelt es sich bei der polemischen Aussage Jesu, dass die

68 Siehe hierzu auch J. Zangenberg, „Art. Samaritaner II“, DNP 11 (2001) 2-4, hier 2.

69 Vgl. auch Crown, „Redating“, 18.

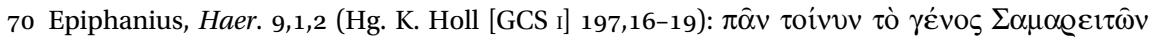

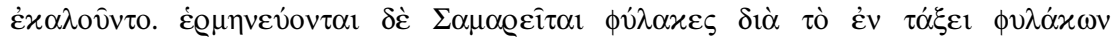

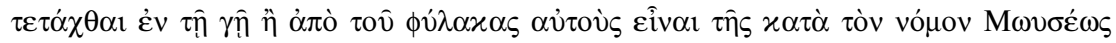

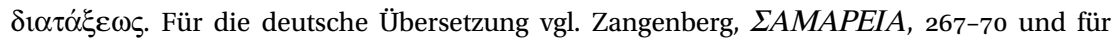
die englische Übersetzung F. Williams, The Panarion of Epiphanius of Salamis: Book I (Sects 1-46) (NHMS 63; Leiden u.a.: Brill, 2009²) 32-3.

71 Zur alleinigen Verwendung des Pentateuch vgl. auch Böhm, Samarien, 78; siehe ferner Crown, „Redating“, 23.

72 Zangenberg, „Samaritaner II“, 3: „Das älteste Dokument samaritan. Theologie und Lit. ist der samaritan. Pentateuch (= SP), eine wohl im 2. Jh. v. Chr. vor allem um Zusätze zum Dekalog erweiterte Rezension des jüd. Pentateuch ..."

73 Siehe hierzu auch B. Becking, „Is There a Samaritan Identity in the Earliest Documents?“, Die Samaritaner und die Bibel, 51-65, hier 65: „This implies that after a period of concordance two competing Yahwistic identities arose: one in Yehud and one in the North."

74 Vgl. Joh 8.37-45.

75 Dass Jesus dann im Johannesevangelium seitens der Juden als „Samaritaner“ bezeichnet wird, könnte einen potentiellen intratextuellen Verweis von Joh 8.48 auf die gegenständliche Passage darstellen, welche die Frage der Genealogien (und ihrer damit verbundenen Identitätsaussagen bezüglich Juden und Samaritanern) zu einem zentralen Thema macht. 
Juden „Kinder des Teufels seien“ (Joh 8.37ff.), um die Formulierung einer innerjüdischen Polemik, ${ }^{76}$ mit welcher eine gegnerische Gruppe ausgegrenzt und dieser die jüdische Identität abgesprochen werden sollte. ${ }^{77}$ Die Beziehung zwischen den beiden Passagen wird sicher auch dadurch verstärkt, dass die Frage der

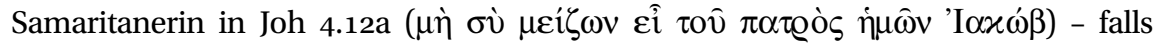
man den dort verwendeten Namen austauscht - wörtlich der Frage der Juden in

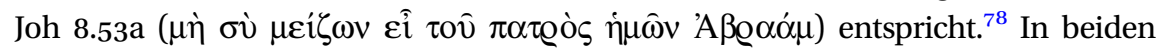
Fällen wird die Identität durch die Genealogie zum Ausdruck gebracht.

Durch die Erwähnung des Feldes, das „Jakob seinem Sohn Josef gab, wo der Brunnen des Jakob war“ (Joh 4.5), und der Bezugnahme auf „unseren Vater Jakob, der uns diesen Brunnen gegeben hat" (Joh 4.12), wird auf der narrativen Ebene die Selbstdefinition der Samaritaner anerkannt. „Sie sehen sich als direkte Nachfahren der Söhne Josephs und Enkel Jakobs, Ephraim und Manasse, an, und setzen den Ursprung ihrer Geschichte mit der Landnahme der zwölf Stämme im Heiligen Land an. “79 Gerade wenn der Evangelist „über die Ortslage und auch über den Konflikt zwischen Samaritanern und Juden relativ präzise Bescheid weiß" ", muss ihm bewusst gewesen sein, dass diese Berufung der Samaritanerin auf den gemeinsamen Vater Jakob in jüdischer Perspektive als klare Aussage einer Zugehörigkeit zum Haus Josef und zum Volk Israel gewertet werden muss. ${ }^{81}$

\subsection{Die potentielle Wiedervereinigung von Juda und dem Haus Josef durch einen davidischen Herrscher}

Dass der halachische Ausschluss der Samaritaner als Teil jüdischer Identitätsbildung gesehen werden muss, ist offensichtlich. ${ }^{82}$ Dieser machte

76 Vgl. U. C. von Wahlde, „,You are of your Father the Devil' in its Context: Stereotyped Apocalyptic Polemic in John 8:38-47“, Anti-Judaism and the Fourth Gospel: Papers of the Leuven Colloquium 2000 (Hg. B. Bieringer/D. Pellefeyt/F. Vandecasteele-Vanneuville; Assen: Van Gorcum, 2001) 418-44, hier 443: „The author is not saying that the Jewish perspective is wrong; he is saying that his opponents do not have the true Jewish perspective!"

77 Von Wahlde, „,YYou are of your Father the Devil'“, 440: „... the polemic itself cannot be said to be inherently anti-Jewish. Indeed, it is precisely a Jewish invention. Rather than being antiJewish, the polemic was ,anti-opposition'.“

$78 \mathrm{Zu}$ diesen beiden Aussagen vgl. auch I. Hjelm, The Samaritans and Early Judaism: A Literary Analysis (JSOT.S 303; Sheffield: Sheffield Academic, 2000) 125.

79 U. Schattner-Rieser, „Prä-, Proto- und Antisamaritanisches in den Qumrantexten“, Qumran aktuell: Texte und Themen der Schriften vom Toten Meer (BThSt 120; Hg. S. Beyerle/J. Frey; Neukirchen: Neukirchener, 2011) 67-109, hier 71. Siehe auch M. Böhm, „Wer gehörte in hellenistisch-römischer Zeit $\mathrm{zu}$,Israel'? Historische Voraussetzungen für eine veränderte Perspektive auf neutestamentliche Texte“, Die Samaritaner und die Bibel, 181-202, hier 199.

80 Frey, „,Gute' Samaritaner?“, 222.

81 Und nach Crown, „Redating“, 50, gilt für das 1. Jhdt. n. Chr., dass sie als Juden anzusehen sind. 82 Vgl. auch M. Lavee, „The Samaritan may be included - Another Look at the Samaritan in Talmudic Literature“, Samaritans: Past and Present. Current Studies (SJ 53/StSam 5; Hg. M. Mor/F. V. Reiterer; Berlin: De Gruyter, 2010) 147-73, hier besonders 170-1. 
jedoch ein positives Verständnis der Samaritaner als „Israeliten“ schwierig. ${ }^{83}$ Dies ändert jedoch nichts daran, dass in prophetischer Literatur „Israel“ sehr wohl die Reste aller Stämme umfassen konnte, ${ }^{84}$ während Texte des Talmud auch mit Rückgriff auf die Geschichte Israels die Samaritaner nicht zu „Israel“ rechnen. ${ }^{85}$ Im Propheten Ezechiel (37.15-28) wird die Wiedervereinigung des Hauses Juda mit dem Haus Josef angekündigt - und zwar unter „David“" ${ }^{86}$ Das Bild, das dafür gewählt wird, ist das von zwei Hölzern die wiedervereinigt werden: „So sprich zu ihnen: So spricht Gott der Herr: Siehe, ich will das Holz Josefs, das in der Hand Ephraims ist, nehmen samt den Stämmen Israels, die sich zu ihm halten, und will sie zu dem Holz Judas tun und ein Holz daraus machen, und sie sollen eins sein in meiner Hand. ${ }^{187}$ Dies scheint auch der Standpunkt anderer normativer Texte des Judentums zu sein. ${ }^{88}$ Interessant ist sicherlich in diesem Zusammenhang, dass die abschließenden Worte des rabbinischen Traktates über die Kutim ${ }^{89}$ (bereits diese Bezeichnung für die Samaritaner stellt

83 Für eine ambivalente Haltung vgl. auch $b$. Qidd. 75b-76a; Rabbi Eleazar und Rabbi Ishmael bezeichnen die „Kutim“ als Proselyten; Rabbi Simeon b. Gamaliel betont, dass die „Kutim“ das Gesetz aufmerksamer befolgen würden als die Israeliten.

84 Vgl. G. N. Knoppers, „Did Jacob Become Judah? The Configuration of Israel's Restoration in Deutero-Isaiah“, Samaria, Samarians, Samaritans: Studies on Bible, History and Linguistics (SJ 66/StSam 6; Hg. J. Zsengellér; Berlin: De Gruyter, 2011) 39-67, hier 6o: „... one important historical trajectory leads to characterizations of Israel (among the Judean elite) that center on Judah itself, the Judean exiles, or some other group within Judah ... Alongside such a narrow trajectory, there is another that begins to define Israelite identity (or Judean identity) along religious lines (e.g., Israel as the people of God) and not simply on the basis of genealogy. Yet another trajectory retains traditional definitions of Israelite identity as encapsulating surviving descendants of all the Israelite tribes."

85 B. Sanh. 21b; dort steht „Israel“ als geschlossene Größe den „Ungebildeten“ (הֶָדוֹ) gegenüber (diese werden in der Antwort von R. Hisda als „Kutim“ bezeichnet und dort im Kommentar als Samaritaner identifiziert).

86 Vgl. hierzu auch P. Stenhouse, „The Chronicle of Abūb 'l-Fath and Samaritan Origins: 2 Kings, 2 Chronicles and Ezra-Nehemiah Viewed through the Prism of Samaritan Tradition“, Die Samaritaner und die Bibel, 303-21, hier 309.

87 Ez 37.19.

88 Vgl. S. Japhet, The Ideology of the Book of Chronicles and Its Place in Biblical Thought (BEAT 9; Frankfurt u.a.: Lang, 1989) 333-4: „Even without approaching the historical question of the status, cohesiveness, and singularity of the Samaritan community at that time, it may be stated that the Chronicler did not consider the Samaritans a separate community. The inhabitants of Samaria were, along with the ,resident aliens,' descendants of Israelite tribes, the Judeans' brothers and an organic part of the people of Israel. We may say that the Chronicler calls for an end to tension and hatred between segments of the people and summons all Israel to unite in worshipping YHWH in Jerusalem.“

89 Zur Verwendung von כָּ in rabbinischen Quellen als Bezeichnung der Samaritaner vgl. auch P. Artzi, „Art. Cuth, Cuthah“, Encyclopaedia Judaica, Bd. v $\left(2007^{2}\right)$ 344-5; zum Traktat Kutim vgl. auch A. Lehnardt, „Das außerkanonische Talmud-Traktat Kutim (Samaritaner) in der innerrabbinischen Überlieferung“, Frankfurter Judaistische Beiträge 26 (1999) 111-23. 
eine implizite Polemik dar) ${ }^{90}$ eine Abkehr von der Verehrung auf dem Berg Garizim und eine Rückkehr zum Jerusalemer Kult als Vorbedingung einer Anerkennung der Samaritaner als Israeliten nennen. ${ }^{91}$ Und damit thematisiert die im Verlauf der Begegnung am Jakobsbrunnen $\mathrm{zu}$ findende Frage, an welchem Ort angebetet werden müsse, ${ }^{92}$ einen nach dem Traktat Kutim zentralen Punkt der Differenzen zwischen Juden und Samaritanern. ${ }^{93}$ „Im Gespräch mit der Samaritanerin stellt Jesus eine neue Verehrung Gottes in Aussicht, in welcher der Streit um die beiden Kultorte und der verfeindeten Religionsparteien überwunden sein wird ... Im johanneischen Text und für das Verständnis der johanneischen Adressaten ist dies die Verehrung des Gottes Israels ... Der 'Streit der Parteien' wird insofern von Jesus selbst überwunden und gerade darin erweist er sich als derjenige, der die eschatologische Erfüllung nicht nur ankündigt, sondern selbst verkörpert. ${ }^{94}$ Diese Beschreibung des Gesprächs zwischen Jesus und der Samaritanerin durch Jörg Frey könnte man durchaus auch als Hoffnung auf eine Wiedervereinigung der Samaritaner mit den Juden deuten, welche Teil prophetischer Hoffnung war, ${ }^{95}$ und als solche auch im

90 Vgl. Jos. Ant. 9,288; zur Bezeichnung Kutim/Cutheans für die Samaritaner vgl. auch G. N. Knoppers, „Samaritan Conceptions of Jewish Origins and Jewish Conceptions of Samaritan Origins: Any Common Ground?“, Die Samaritaner und die Bibel, 81-118, hier 115: „In later Jewish traditions, these deportees, named ,Cutheans' after the name of one of the five foreign ethnic groups imported into the land, are construed as the ancestors of the Samaritans.“ Vgl. ferner A. Lehnardt, „Die Taube auf dem Garizim: Zur antisamaritanischen Polemik in der rabbinischen Literatur", Die Samaritaner und die Bibel, 285-302, hier 288. Siehe ferner Kartveit, The Origins of the Samaritans, 360: „Their claim to being 'Israel' or 'Joseph' is countered by accusations of living in mixed marriages, of having a foreign origin and an illegitimate temple site, in short of being 'enemies' and 'fools'."

91 Kutim 2,8 (M. Higger, Seven Minor Treatises [New York: Bloch, 1930] 46): „When shall we receive the Samaritans? When they renounce Mount Gerizim and acknowledge Jerusalem and the resurrection of the dead." Vgl. auch P. W. van der Horst, "Anti-Samaritan Propaganda in Early Judaism“, Persuasion and Dissuasion in Early Christianity: Ancient Judaism, and Hellenism (Contributions to Biblical Exegesis 33; Hg. P. W. van der Horst; Leuven: Peeters, 2003) 25-44, hier 26 Anm. 4: „It is to be borne in mind that the closing paragraph of the same tractate says that Samaritans may only be readmitted into the Jewish community 'when they have renounced Mount Gerizim and acknowledged Jerusalem and the resurrection from the dead', which of course implies that without that they are complete outsiders."

92 Vgl. Joh 4.20-4.

93 Vgl. auch B. Hall, „From John Hyrcanus to Baba Rabbah“, The Samaritans (Hg. A. D. Crown; Tübingen: Mohr, 1989) 32-54, hier 40: „... we may be confident, on the basis of the relevant material in the Fourth Gospel (cf. John 4:21-22) and the writings of Josephus (cf. $A J$ Ix. 290), that in the first century AD at least the vast majority of Samaritans worshipped the same God as the Jews worshipped, and worshipped this God as their sole God."

94 Frey, „,Gute' Samaritaner?", 228-9.

95 Vgl. auch K. Schmid, „Die Samaritaner und die Judäer: Die biblische Diskussion um ihr Verhältnis in Josua 24“, Die Samaritaner und die Bibel, 31-49, hier 49: „Jos 24 steht damit 
Johannesevangelium zum Ausdruck kommt. ${ }^{96}$ In diesem Kontext kann die Anbetung in "Geist und Wahrheit" (Joh 4.24) anstelle „auf diesem Berg oder in Jerusalem" (Joh 4.21) als Überwindung des Streits zwischen Juden und Samaritanern um den rechten Ort der Anbetung gewertet werden. ${ }^{97}$

\section{Ergebnis}

Die Schilderung der Samaritanerin in Joh 4 ist weit positiver als bisher wahrgenommen; implizit wird sogar eine Aussage über ihre Zugehörigkeit zu Israel getroffen. ${ }^{98}$ Deswegen greift selbst die relativ positive Bewertung der Passage durch Martina Böhm zu kurz: „Bei Joh 4 fällt dabei auf, daß die Erzählung eher um reale theologische Differenzen zwischen Juden und Samaritanern im 1. Jh.n. kreist, als daß sie Teil hat an zeitgenössischer Polemik und Vorurteilen, die durch die Versionen von 2Kön 17 geprägt worden sein könnten. Insofern dürfte der Text als ein Beispiel für eine ,neutrale' Schilderung der Samaritaner stehen. “99

Es handelt sich nicht einfach um eine „neutrale“ Darstellung. Vielmehr darf sich die Samaritanerin durch die von Jesus nicht in Frage gestellte Berufung auf ihre Abstammung von „unserem Vater Jakob“ stellvertretend für alle Samaritaner als vollwertiges Mitglied eines der Stämme Israels bezeichnen. ${ }^{100}$ Die Tatsache, dass der Brunnen in dem Gebiet liegt, „das Jakob seinem Sohn Josef gegeben hatte“, zeigt ferner, dass die Samaritaner im Johannesevangelium

im Verein mit verschiedenen nachexilischen Texten namentlich aus der prophetischen Überlieferung, die wohl seit der Exilszeit Hoffnungen auf eine Restitution ganz Israels hegen: Jer 30,3.8f.; 31,27f.; 31ff.; Ez 34,23ff.; 37,15-28; Ob 18ff.; Jes 11,11-16; Jer 3,18; Sach 9,9ff.; 10,6ff.“

96 Vgl. Hjelm, Samaritans, 120: „John 4,1-42's participation in the dialogue about JudaeanSamaritan relationship restores the Old Testament prophecy that 'there shall be one flock and one shepherd' (Jn 10.16; Ezek. 34.17-31; 37.16-28).“

97 Siehe hierzu auch Magen, Samaritans, 66: „The main dispute between the Samaritans and the Jews concerned the chosen place and the location of the temple, whether in Jerusalem or at Mt. Gerizim.“

98 Dagegen jedoch Frey, „,Gute‘ Samaritaner?“, 232: „Auch bei ... Johannes ist keine präzise Einordnung der Samaritaner in ein Stämme-Schema zu erkennen ...“

99 Böhm, Samarien, 144-5.

100 Vgl. auch R. Pummer, „Samaritanism - A Jewish Sect or an Independent Form of Yahwism?“, Samaritans: Past and Present, 1-24, hier 17: „... the Samaritans' self-understanding as Israelites is a central tenet of their faith." Siehe ferner E. Nodet, „Israelites, Samaritans, Temples, Jews“, Samaria, Samarians, Samaritans, 121-71, hier 121: „This study aims at showing that the Samaritans of Shechem are the heirs of the early Israelites, and not a downgraded Jewish sect as old Judean traditions and many modern scholars claim.“ 
korrekt dem „Haus Josef ${ }^{\text {“101 }}$ und damit den Stämmen Ephraim und Manasse zugeordnet werden. Auch an anderen Stellen dieser Passage des Johannesevangeliums scheint ein großes Wissen um samaritanische Theologie vorausgesetzt zu werden. ${ }^{102}$

Ferner wird durch die pointierte Darstellung der Samaritanerin als in einer nicht der Tora entsprechenden Beziehung lebend, die intratextuell mit den Juden kontrastiert wird, denen Jesus die Abrahamskindschaft abspricht, eine eindeutige theologische Aussage bezüglich der Samaritaner gemacht. Dies wird dadurch unterstrichen, dass die theologische Bedeutung der Frage nach einer „Herkunft aus dem Ehebruch“ im Rahmen der Auseinandersetzung um die Herkunft der Juden von Abraham dezidiert thematisiert wird. ${ }^{103}$ Eine samaritanische Frau, die nach fünf Ehen ${ }^{104}$ mit einem Mann, „der nicht ihr Mann ist“, lebt, ${ }^{105}$ darf durch ihre Berufung auf den Erzvater Jakob ihre Zugehörigkeit zum Volk Israel unwidersprochen feststellen. ${ }^{106}$

101 So auch Jos. Ant. 9,291.

102 So Kartveit, The Origins of the Samaritans, 315: „The Johannine understanding in John 4:25 might be on the right track ... Such messianic interpretation in the Gospel of John may be not too far away from the - admittedly much later - attestation of the Samaritan Taheb in the Durran. The name Taheb is not inconceivable as a Messianic title ..."

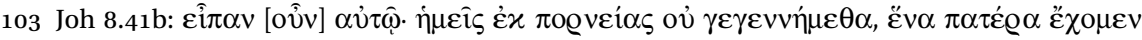

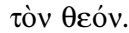

104 Vgl. hierzu auch Barrett, St. John, 235: „It is to be noted that the Rabbis did not approve of more than three marriages, though any number was legally admissible (S.B. II, 437). It does not seem probable that John's intention is to show that, though both are now transcended, the Samaritans are morally inferior to the Jews. In fact, it is quite possible, and may well be right, to take these words as a simple statement of fact, and an instance of the supernatural knowledge of Jesus (cf. 1.48); this view is supported by v. 29 ( $\pi \dot{\alpha} v \tau \alpha \ddot{\alpha}$

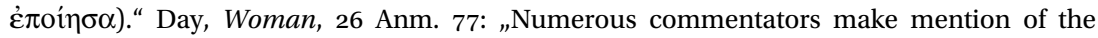
Jewish sanction against more than three marriages, but as Pheme Perkins, The Gospel according to St. John: A Theological Commentary (Chicago: Franciscan Herald Press, 1988), 55, points out, 'The Samaritan legal situation is unknown (Jewish law permitted three wives); so it is difficult to tell whether Jesus' description of the woman's husbands implies condemnation or is a demonstration that he has unusual knowledge of the woman's personal life.'

105 Der eheliche Bund wird biblisch auch als Metapher für die Beziehung Gottes zu seinem Volk verwendet; die Tatsache, dass sie mit einem Mann zusammenlebt, der nicht „ihr“ Mann ist, würde wohl im biblischen Sinn als $\pi$ o@veí $\alpha$ gewertet, während ihr die Zugehörigkeit zu den Söhnen und Töchtern Jakobs im vorliegenden Text nicht abgesprochen wird. Und damit handelt es sich nach der Definition von Amit (vgl. oben) zumindest bei der Frage der Männer der Samaritanerin eindeutig um eine verschleierte Polemik gegen die Juden und ihre Berufung auf die Abstammung von Abraham (vgl. Joh 8.37-45).

106 Gerade die Frage der Zugehörigkeit wird im Rahmen der Symbolhandlung des Propheten Hosea (die ja eben mit der Frage der ehelichen Treue verbunden ist) in Hosea 1.2 beschrieben: Die Heirat mit der käuflichen Frau steht zeichenhaft für die Aussage „nicht mein Volk“

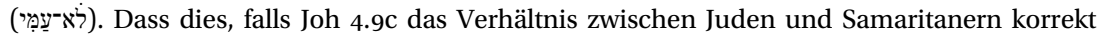
beschreibt, trotz der problematischen persönlichen Umstände der Samaritanerin nicht 
Der Verfasser des Textes wählt durch die verwendete Genealogie eine Kodierung, die in besonderer Weise jüdischen Lesern verständlich ist. Dies zeigt, wie sehr der Verfasser in jüdischen Traditionen verwurzelt war, während gleichzeitig ein erstaunlich positives Bild der samaritanischen Identität gezeichnet wird. ${ }^{107}$ Es ist also nicht mehr möglich, unter Verweis auf Joh $4.9 \mathrm{zu}$ behaupten, dass sich generell im Neuen Testament eine ablehnende Haltung gegenüber den Samaritanern belegen ließe. ${ }^{108}$

Vielmehr sollte die Begegnung von Jesus mit der Frau am Brunnen als ein Beleg dafür gewertet werden, dass die Samaritaner sich selbst um die Mitte des ersten nachchristlichen Jahrhunderts als Nachkommen des Hauses Josef - mit den Stämmen Ephraim und Manasse - ansahen und dass johanneische Christen, die selbst einen „Messias“" ${ }^{\text {109 }}$ verehrten, den sie auch als den „Gesalbten“ ( $\chi \varrho 1 \sigma \tau o ́ \varsigma){ }^{110}$ bezeichneten, und zu denen zweifellos zur Zeit der Abfassung des Johannesevangeliums auch zahlreiche Juden-Christen gehörten, welche die genealogische Argumentation des vorliegenden Textes verstanden, diese Samaritaner implizit als Teil des Volkes Israel anerkannten. ${ }^{111}$ Damit ist die traditionelle Auslegung dieser Passage als Grundlegung von „Heidenmission“ und weltweiter Verkündigung des Christentums Teil christlicher Identität, der im Gegensatz zum Judentum genealogische Argumente fremd sind: „Hier ist nicht Jude noch Grieche, hier ist nicht Sklave noch Freier, hier ist nicht Mann noch Frau; denn ihr seid allesamt einer in Christus Jesus. ${ }^{112}$ Die hier - und an vielen anderen Stellen neutestamentlicher, vor allem paulinischer, Schriften zu findende Entgrenzung des „Volkes Gottes“ im Sinne „aller Gläubigen“ aus

thematisiert wird, sondern vielmehr das Gegenteil durch den Text zum Ausdruck gebracht wird, darf als auffällig bezeichnet werden.

107 Matassa, „Art. Samaritans“, 719: „... the origins and early history of the Samaritans are quite problematic because the sources are far removed from the events and because the nonSamaritan sources tend to be hostile."

108 E. Friedheim, „Some Notes about the Samaritans and the Rabbinic Class at the Crossroads“, Samaritans: Past and Present, 193-202, hier 194: „The New Testament similarly reflects the inimical state of Jewish-Samaritan relations." Vgl. hierzu z. B. die Haltung von Flavius Josephus (Jos. Ant. 11,341), welcher die Samaritaner nicht als mit den Juden verwandt ansah.

109 Vgl. Joh 1.41 und 4.52.

110 Joh 1.17, 20, 25, 41 etc.

111 Vgl. R. J. Coggins, Samaritans and Jews: The Origins of Samaritanism Reconsidered (Oxford: Blackwell, 1975) 164: ,... all the evidence suggests that the decisive formative period for Samaritanism was the epoch from the third century вс to the beginning of the Christian era; and that it emerged from the matrix of Judaism during this time, with some measure of communication continuing well into the Christian era between Samaritans and various Jewish groups."

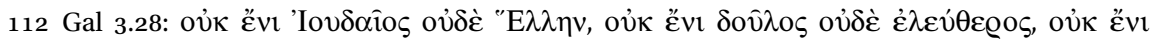

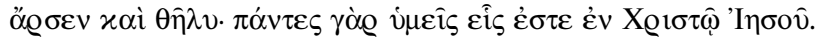


„allen Völkern“ ist, wie anhand der impliziten genealogischen Argumente gezeigt wurde, in der vorliegenden Stelle des Johannesevangeliums nicht zu finden. Ferner muss aufgrund der vorliegenden Analyse die Hypothese abgelehnt werden, dass es sich bei den Samaritanern aus Sicht des Johannesevangliums um „,halbe Heiden'“ gehandelt habe, „die hier stellvertretend für alle Heiden stehen“. ${ }^{113}$

Und damit ist die vorliegende Stelle als eine der Passagen zu werten, in denen die frühchristliche Identität eine (zumindest zu Teilen) judenchristliche Identität war, bei der es erst einmal um die Frage ging, wer „ein wahrer Israelit“ (Joh 1.47b) sei - schließlich gab es im ersten Jahrhundert auch die heiß umkämpfte Frage, ob die Beschneidung als Zeichen der Zugehörigkeit zum Volk Gottes auch für Christen gelte (vgl. Apg 15).

German abstract: In der Erzählung von der Begegnung zwischen Jesus und der namenlosen Samaritanerin (Joh 4.4-42) wird davon berichtet, dass „der Brunnen Jakobs“ dessen Sohn Josef gegeben worden sei (Joh 4.5). Indem die Samaritanerin Jakob als "unseren Vater" (Joh 4.12) bezeichnet, erhebt sie für sich und die Samaritaner den Anspruch, „Kinder Israels“ zu sein. Die Samaritaner werden also ausdrücklich als Angehörige der Stämme Ephraim und Manasse bezeichnet, die zusammen das „Haus Josefs“ bilden. Diese Genealogie ist in ein Motiv der Schrift eingebettet: Die Begegnung am Brunnen. Deshalb muss die Frage aufgeworfen werden, ob diese Passage tatsächlich vorrangig als „Mission unter Nicht-Juden“ interpretiert werden darf. Schließlich scheint die Erzählung sehr deutlich die Samaritaner als „Kinder Israels“ zu thematisieren.

113 Hengel, Die johanneische Frage, 301. So ähnlich auch Schulz, Johannes, 74: „Die Samaritaner waren ein halb heidnisches Mischvolk, das sich aus den im Land verbliebenen Bewohnern des im Jahre 722 zerstörten Reiches Israel und assyrischen Kolonisten zusammensetzte (vgl. 2.Kön. 17,24-41). Trotzdem wollten sie echte Israeliten sein, auch wenn sie den judäischen Juden als halbe Heiden galten. “ Siehe auch Frey, „,Gute' Samaritaner?“, 223-4: „Sie stehen somit gerade für den Universalismus der Botschaft von Jesus, der Schranken überwindet ... In diesem übergreifenden Horizont fungieren die Samaritaner in Joh 4 bereits als die erste Vorhut der Heidenwelt, die später ... kommen und am Heil teilhaben soll." 